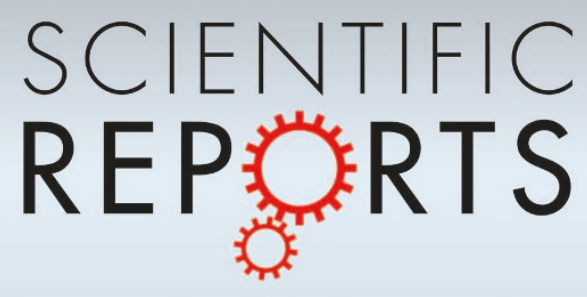

OPEN

SUBJECT AREAS:

GELS AND HYDROGELS

BIOMEDICAL MATERIALS

MOLECULAR SELF-ASSEMBLY

Received

9 June 2014

Accepted

21 November 2014

Published

13 January 2015

Correspondence and requests for materials should be addressed to

J.G. (chemgaojie@ nankai.edu.cn) or J.H. (haojihui@tjmuch.com)

\section{Zinc-Triggered Hydrogelation of Self-assembled Small Molecules to Inhibit Bacterial Growth}

\author{
Chao Xu', Yanbin $\mathrm{Cai}^{2,3}$, Chunhua Ren ${ }^{2,3}$, Jie Gao ${ }^{3}$ \& Jihui Hao'
}

\begin{abstract}
${ }^{1}$ National Clinical Research Center for Cancer, Key Laboratory of Cancer Prevention and Therapy, Department of Pancreatic Cancer, Tianjin Medical University Cancer Institute and Hospital, Tianjin, P. R. China, ${ }^{2}$ College of Life Science, Nankai University, Tianjin 300071, P. R. China, ${ }^{3}$ State Key Laboratory of Medicinal Chemical Biology and Collaborative Innovation Center of Chemical Science and Engineering (Tianjin), Nankai University, Tianjin 300071, P. R. China.
\end{abstract}

There is a significant need to develop antibacterial materials that could be applied locally and directly to the places surrounded by large amount of bacteria, in order to address the problems of bacterial antibiotic-resistance or irreversible biofilm formation. Hydrogels are thought to be suitable candidates due to their versatile applications in biomedical field. Among them, small molecular hydrogels have been paid lots of attention because they are easy to design and fabricate and often sensitive to external stimuli.

Meanwhile, the antibacterial activity of metal ions are attracting more and more attention because resistance to them are not yet found within bacteria. We therefore designed the zinc ion binding peptide of Nap-GFFYGGGHGRGD, who can self-assemble into hydrogels after binds $\mathrm{Zn}^{2+}$ and inhibit the growth of bacteria due to the excellent antibacterial activity of $\mathrm{Zn}^{2+}$. Upon the addition of zinc ions, solutions containing Nap-GFFYGGGHGRGD transformed into supramolecular hydrogels composed of network of long nano-fibers. Bacterial tests revealed an antibacterial effect of the zinc triggered hydrogels on $E$. coli. The studied small molecular hydrogel shows great potential in locally addressing bacterial infections.

M odern medical technology requires sterility as the first and most important principle, yet bacterial infections still cause millions of deaths every year all over the world ${ }^{1-3}$. The combat between human and bacteria escalated mainly due to antibiotic-resistance of bacteria ${ }^{4-6}$. Such resistance has developed on a very large scale over time, greatly reducing the effectiveness of drugs, and is an ever growing problem. Except planktonic bacteria gain resistance to common antibiotics, the reason why infections are difficult to cure also lay in the formation of irreversible biofilm ${ }^{7-9}$, who can act as a kind of defensive barrier to immune system and antibiotics thus resulting in tolerance of bacteria embedded within biofilms to very high concentrations of multiple antibiotic agents. Therefor conventional way of drug using exhibits very poor effect on chronic bacterial infections which often associated with biofilm formation such as Candida albicans (candiasis) ${ }^{10}$, Staphylococcus aureus (chronic rhinosinusitis) ${ }^{11}$ and enteropathogenic Escherichia coli (recurrent urethritis) ${ }^{12,13}$. Drug-resistant bacterial infections result in numerous negative effects upon society such as treatments with higher doses of drugs, longer hospital stays and increased mortality, leading to a stronger desire of new anti-bacterial technologies and materials for medical utility.

To address these problems, new generation of antibiotic agents against drug resistant bacteria have been developed (e.g. antibacterial agents derived from classical common used antibiotics ${ }^{14}$, new generation of peptide antibiotics ${ }^{15}$ and intracellular hydrogelation of small molecules for bacterial growth inhibition $\left.{ }^{16}\right)$. Surprisingly, metal ions have also found applications in lots of advanced antibacterial systems ${ }^{17}$. Metal ions of silver $(\mathrm{Ag})^{18}$, gold $(\mathrm{Au})^{19}$, zinc $(\mathrm{Zn})^{20,21}$, copper $(\mathrm{Cu})^{22}$, titanium $(\mathrm{Ti})^{23}$, magnesium $(\mathrm{Mg})^{24}$, along with their oxides, could kill or inhibit growth of microbes through multiple mechanisms, thereby making development of resistance unlikely.

Meanwhile, alternative way of treatment which can be applied locally and immediately in the surrounding of bacteria to release the antibacterial cargo they are carrying is being developed too ${ }^{15,25}$, in order to address the problem of biofilm-induced bacterial infections. Among all other biomaterials, hydrogels have been extensively explored in biomedical applications such as controlled drug release and delivery ${ }^{26}$, bio-sensing and imaging ${ }^{27}$ and tissue engineering ${ }^{28,29}$ since they can easily adapt to different environments both in vitro and in vivo. Many experimental and clinical studies have shown that hydrogels containing antibacterial agents are very good antimicrobial materials. For example, hydrogels releasing the molecular of NO are very good anti-microbial 


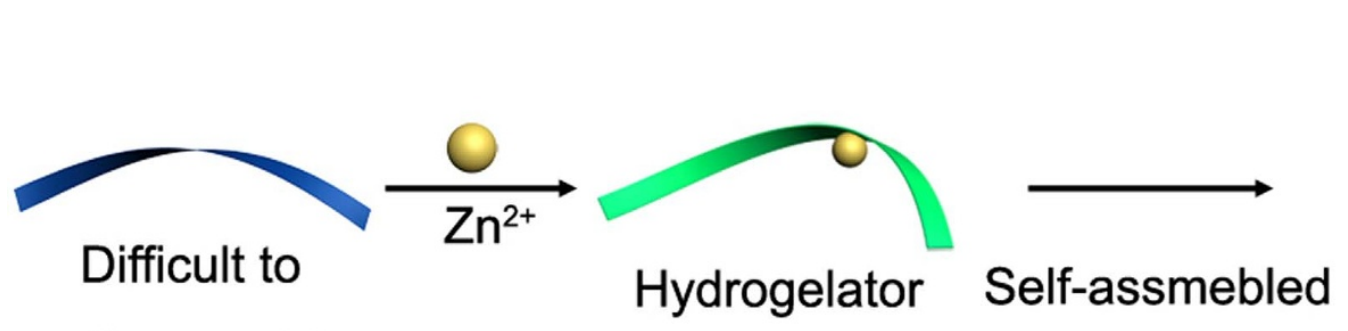

self-assemble

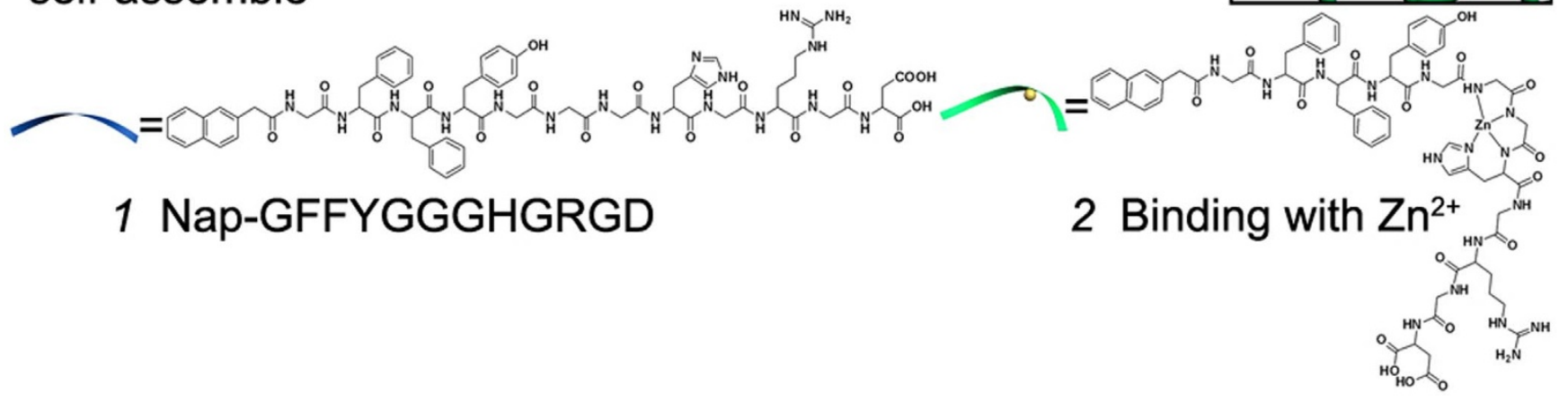

Figure $1 \mid$ Chemical structure of $\mathrm{Zn}^{2+}$ binding peptide and schematic diagram of it binding with $\mathrm{Zn}^{2+}$ and self-assembling into hydrogel. Blue ribbon and green ribbon stand for peptide before and after binding with $\mathrm{Zn}^{2+}$, respectively.

wound dressings $s^{30,31}$, hydrogels containing zinc chloride ${ }^{21,32}$, silver nitrate $^{18}$ or other metal ion nanoparticles ${ }^{33}$ can serve as antimicrobial surface grafts or medical materials, hydrogel microspheres release syn. melittin in a controlled manner to inhibit bacterial growth ${ }^{34}$, et al.

As a kind of newly developed hydrogels, supramolecular hydrogels have attracted broad research interest in recent years due to their inherent properties such as easily controllable of mechanical property, diversity of components ${ }^{35}$ and excellent biocompatibility ${ }^{36,37}$. Supramolecular hydrogels were formed by the self-assembly of small molecules, usually upon the trigger of external stimuli including $\mathrm{pH}^{38-40}$, temperature ${ }^{39}$ or ionic strength changes ${ }^{39,41}$, enzymatic catalysis ${ }^{42-45}$, light irradiation ${ }^{46-49}$, redox-stimuli ${ }^{50-52}$, et al. Recently, several research groups have made progress on metal ion stimulated self-assembly hydrogels. Stupp and co-workers have reported a calcium-triggered peptide hydrogel system for biomimetic hydroxyapatite mineralization in three dimensions ${ }^{53}$. Schneider and coworkers have developed several peptides that can form $\beta$-hairpin structure and hydrogels upon the addition of zinc ion and other heavy metal ions ${ }^{54,55}$. Xu and co-workers have reported peptide hydrogel systems responsive to calcium ions, potassium ion and nickel ion ${ }^{56-58}$. We have developed a barium-triggered peptide hydrogel system to help remove heavy metal from water ${ }^{59}$. In this work, combining the concepts of heavy-metal stimulated self-assembling and antibacterial hydrogels, we designed a zinc ion triggered small molecular hydrogel, who can inhibit the growth of bacteria due to the excellent antibacterial activity of $\mathrm{Zn}^{2+}$.

\section{Results}

Design and Synthesis of the Self-assembled Small Molecule. We firstly designed the chemical structure of self-assembled small molecules Nap-GFFYGGGHGRGD (1) based on commonly used self-assembly peptides in our previous work (the chemical structure of Nap-GFFYGGGHGRGD was shown in Fig. 1). The design of molecular structure was following these principles: (i) Nap-GFFY was used as the motif of self-assembling because it could not only easily self-assemble into hydrogels by itself but also help short peptides containing it to form hydrogels. Its strong self-assembling ability was mainly due to the $\pi$ - $\pi$ stacking effect between aromatic rings and hydrophobic interaction between molecules. (ii) The already known divalent metal ion binding peptide sequence GGH was added as the metal binding site (possible binding model was shown in Fig. 1) ${ }^{60}$. (iii) The sequence of RGD was included for the hope of more hydrophilicity and better biocompatibility. We hypothesized that when peptide 1 binds $\mathrm{Zn}^{2+}$ ions, some of its side chain becomes neutral (as shown in Fig. 1), the hydrophilic peptide becomes more hydrophobic and gain better self-assembly ability, thus hydrogel formation is enabled. The synthesis of NapGFFYGGGHGRGD was easy and straightforward, using already mature solid phase peptide synthesis technology (SPPS, see details in Supplementary Methods).

Metal-triggered Formation of Hydrogels. After pure peptide was obtained by reverse phase high performance liquid chromatography (HPLC), we tested its ability to self-assemble into hydrogels. Although the peptide has the sequence of Nap-GFFY, which was very easy to form hydrogels, the whole structure couldn't able to form homogeneous or stable hydrogel by itself at room temperature (Fig. 2A), neither in PBS nor in water. However, the solution containing high concentration of compound $(1.0 \mathrm{wt} \%$ in $\mathrm{pH}$ 7.4 PBS) became rather viscous and a little bit cloudy, suggesting that the peptide might form some kind of micro-structures in it. We then added different equiv. of $\mathrm{ZnCl}_{2}$ into PBS solutions containing 1.0 wt\% of 1 to see whether $\mathrm{Zn}^{2+}$ could enable the transition from solution to gel and how the amount of zinc ions would affect this process. After the addition of 1.0 equiv. of $\mathrm{Zn}^{2+}$ to abovementioned solutions, we observed a rapid hydrogel formation (within 5 minutes, Fig. 2D). Gradually reduce the $\mathrm{Zn}$ ions added to the solution, solutions on the addition of 0.2 to 0.9 equiv. of $\mathrm{Zn}^{2+}$ also formed self-supporting hydrogels at room temperature (some optical images of the hydrogels was shown in Fig. $2 \mathrm{~B}$ and $\mathrm{C}$ ), though the hydrogelation process was delayed (about 2 hours after addition of 0.2 equiv. of $\mathrm{ZnCl}_{2}$ ). And the minimum equiv. of $\mathrm{Zn}^{2+}$ to induce solution at such condition to form hydrogel was 0.2. These phenomenon clearly demonstrated that compound 1 possessed stronger ability of forming hydrogels after binding with $\mathrm{Zn}^{2+}$ than the peptide it alone. Another thing worth mention was that if we keep the peptide to Zinc ion ratio fixed at $1: 1$, the minimum concentration of peptide to form hydrogel was $0.3 \mathrm{wt} \%$ (details of the minimum hydrogelation concentration can be found as Supplementary Table S1 online). 

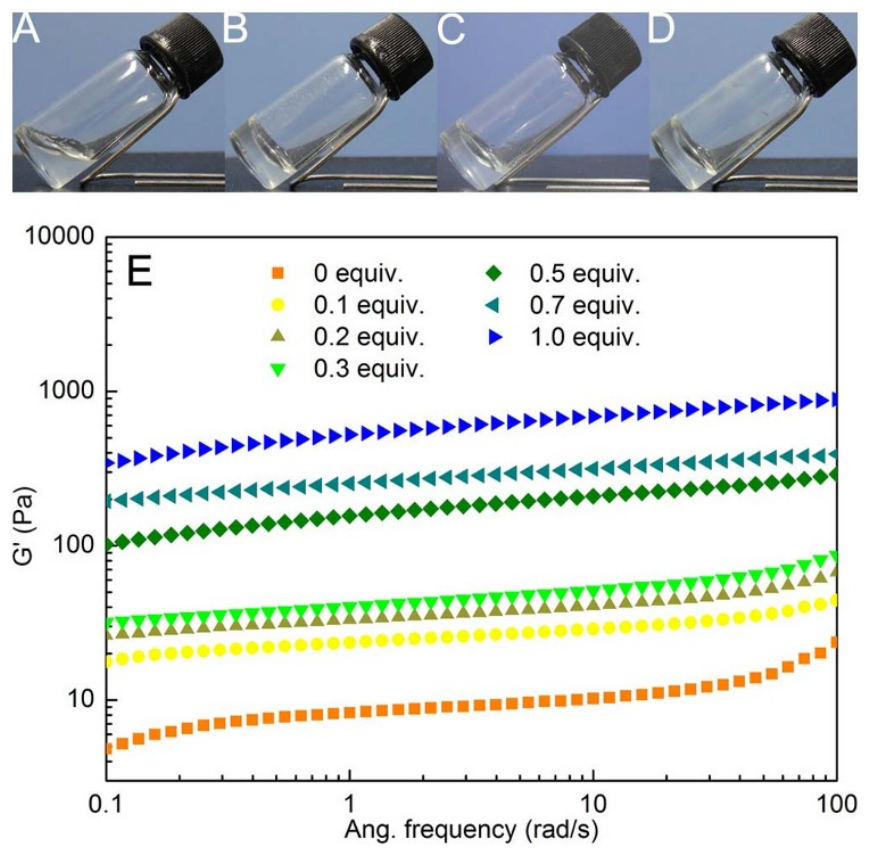

Figure 2 Optical images of solutions and hydrogels and the rheology test results. A)Solution containing $1.0 \mathrm{wt} \%$ of compound and hydrogels formed after adding B) 0.3, C) 0.7 and D) 1.0 equiv. of $\mathrm{Zn}^{2+}$. E) Oscillatory rheological dynamic frequency sweeps of $1 \mathrm{wt} \%$ peptide in $\mathrm{pH} 7.4$ buffer at $25^{\circ} \mathrm{C}$ with addition of different equiv. of $\mathrm{Zn}^{2+}: 0,0.1,0.2,0.30 .5,0.7$ and 1.0, respectively. $\mathrm{G}^{\prime}$ is the storage modulus in units of $\mathrm{Pa}$.

Meanwhile, we eliminate GGH sequence from compound 1 and gain an peptide of Nap-GFFYGRGD. 1.0 equiv. of Zinc ions were added to solution containing $1.0 \mathrm{wt} \%$ of Nap-GFFYGRGD and it was still solution (see Supplementary Fig. S5 A and B online), demonstrating the importance of GGH sequence for zinc ion triggered hydrogelation. We also tested other divalent metal ions including $\mathrm{Ca}^{2+}, \mathrm{Mg}^{2+}, \mathrm{Ba}^{2+}, \mathrm{Mn}^{2+}$ and $\mathrm{Sr}^{2+}$, and found that the addition of 1 equiv. of $\mathrm{Ca}^{2+}$ and $\mathrm{Mg}^{2+}$ could also lead to hydrogelation, while other metal ions could not lead to gelation (see Supplementary Fig. S5 C-G online).

In order to further clarify the interaction between compound 1 and zinc ions, we tested the zeta potential of the nanostructures in solution containing compound 1 and hydrogels triggered by different amount of zinc ions. Results indicated that the nanostructures gradually changed from negative charged to positive charged with the increasing amount of $\mathrm{Zn}^{2+}$ (Fig. S7).

Mechanical Properties. As illustrated in Fig. 1, one equiv. of peptides could bind the same mole of zinc ions and then transformed into hydrogelator 2 and self-assembled into hydrogel. Thus, we hypothesized that hydrogels induced by less amount of $\mathrm{Zn}^{2+}$ should have less hydrogelators and leading to poorer mechanical property than the one induced by more $\mathrm{Zn}^{2+}$. Therefore we used a rheometer to study the mechanical properties of solution containing $1.0 \mathrm{wt} \%$ of compound 1 and hydrogels formed by treating it with different equiv. of $\mathrm{Zn}^{2+}$. As shown in Fig. 2E, before the addition of zinc ions, the solution of peptide possessed the lowest storage modulus $\left(G^{\prime}\right)$ value of around 8.2 Pa. By increasing the amount of zinc ions in the solution to $0.1,0.2,0.3,0.5,0.7$ and 1.0 equiv., the according G' value gradually increased to about 23.5, 33.2, 40.3, 156.3, 253.6 and $525.5 \mathrm{~Pa}$, respectively. These results have proved that more $\mathrm{Zn}^{2+}$ would lead to stronger hydrogels. Since the differences in mechanical property were macroscopically reflections of microstructural diversity in the hydrogels, we next used transmission electron microscope (TEM) to reveal the morphology of the solution and hydrogels.
Micro-structure of the Solution and Hydrogels. TEM images of solution and hydrogels containing $1.0 \mathrm{wt} \%$ of 1 were shown in Fig. 3. Most of the structures formed by compound 1 in PBS solution were random aggregations of very short fibrous like structures (shown in Fig. 3A, indicated by black arrows) in the size of about $351 \mathrm{~nm} \times 234 \mathrm{~nm}$. We have also noticed that a few longer fibers were formed and cross-linked with each other (shown in Fig. 1A, indicated by white arrow), however these fibers still not long enough to form cross-linked network all over the hydrogel, which is a key factor to form self-supporting hydrogels. In hydrogels formed by addition of $0.3,0.7$ and 1.0 equiv. of $\mathrm{Zn}^{2+}$, it was clear in the TEM images (Fig. 3B,C and D) that much longer fibers (with lengths longer than $5.2 \mathrm{~mm}$ ) have formed and entangled with each other all over the hydrogel. An obvious phenomenon was that by adding more $\mathrm{Zn}^{2+}$ to the solution, the diameter of the fibers became larger. For the hydrogel containing 0.3 equiv. of zinc ions, the diameter of fibers was $48.5 \pm 7.6 \mathrm{~nm}$, and it increased to $67.8 \pm$ $4.6 \mathrm{~nm}$ and $96.3 \pm 7.5 \mathrm{~nm}$ for the gel containing 0.7 and 1.0 equiv. of zinc ions. These results agreed with the observations of hydrogel formation and indicated that addition of zinc ions could lead to fibril network formation, enhancement of G' value, and the formation of hydrogels.

Bacterial Inhibition Activity tests. Having shown that zinc ions could trigger the hydrogelation of compound 1, the antibacterial activity of these hydrogels were tested on E. coli. We first cultured the bacteria within and on the surface of the hydrogels (concentration of compound 1 was $1.0 \mathrm{wt} \%$ ). We monitored their growth within hydrogels containing different amount of zinc ions as changes in absorbance at $600 \mathrm{~nm}$ after 6 hours (Fig. 4). Compared to the original absorbance value, the one of the control group (bacteria in LB medium without addition of $\mathrm{Zn}^{2+}$ ) have grown to about 10 times after 6 hours' culturing. For hydrogels triggered by 0.1 to 0.5 equiv. of zinc ions, being inversely proportional to the amount of zinc ions, the absorbance growth of bacteria ranged from 7 times to about 1.5 times of the original one. For hydrogels triggered by more than 0.5 equiv. of zinc ions, the growth of bacteria were almost $100 \%$ inhibited because the absorbance remained the same. Bacteria did not form any colony visible to the naked eye on surface of zinc ion triggered hydrogel (see Supplementary Fig. S9 online) but had grown into several colonies on surface of agarose and supramolecular hydrogels bearing no GGH sequence (NapFFGRGD). These results clearly demonstrated that the zinc triggered hydrogels can inhibit the growth of $E$. coli both within and on the surface of the hydrogel, and its antibacterial activity strongly depends on the amount of zinc ions it contains.

To better understand the effect of the zinc triggered hydrogel, bacteria were incubated within $\mathrm{ZnCl}_{2}$ containing $\mathrm{LB}$ medium and mixture of zinc ions and above mentioned supramolecular hydrogels (Nap-FFGRGD). According to Fig. 4, the final absorbance of $\mathrm{Zn}^{2+}$ containing medium is higher than hydrogel triggered by same amount of zinc ions. Similarly, more bacterial growth was inhibited by zinc ion binding hydrogels than mixture of same amount of zinc ions and hydrogels formed by Nap-FFGRGD (see Supplementary Fig. S10 online). Both results seems to indicate that zinc triggered hydrogel is more effective than expected compared with the same concentration of non-encapsulated $\mathrm{Zn}^{2+}$.

Finally, we tested the antibacterial ability of compound 1 itself to see if it has any possible toxicity on E. coli. Results (see Supplementary Fig. S11 online) shows that E. coli in solutions containing different amount of 1 have grown as much as in the LB medium, thus demonstrating that the peptide itself has no innate bactericidal activity.

The antibacterial ability of zinc ion triggered hydrogel comes from zinc ions it contains, and the bind zinc ions should release from the hydrogel to accomplish the inhibiting task. Hence the zinc ion release 

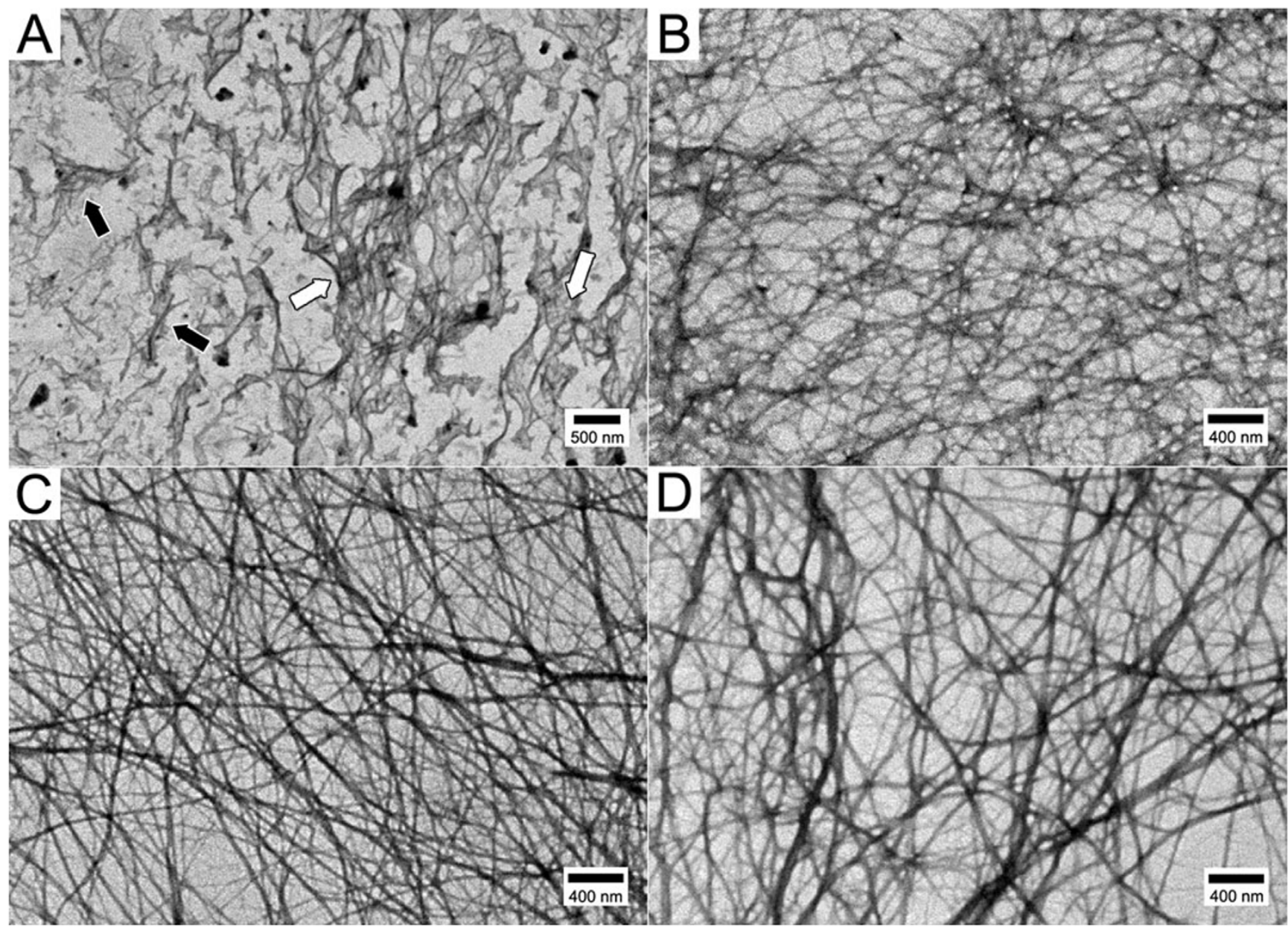

Figure 3 TEM images of (A) solution of Nap-GFFYGGGHGRGD (1.0 wt\% in PBS), hydrogels of Nap-GFFYGGGHGRGD (1.0 wt\% in PBS) with addition of (B) 0.3 , (C) 0.7 and (D) 1.0 equiv. of $\mathrm{Zn}^{2+}$.

profile was measured by ICP-AES, and results (see Supplementary Fig. S12 online) indicated that zinc continuously release into the supernatant during following 6 hours and no boost release was seen.

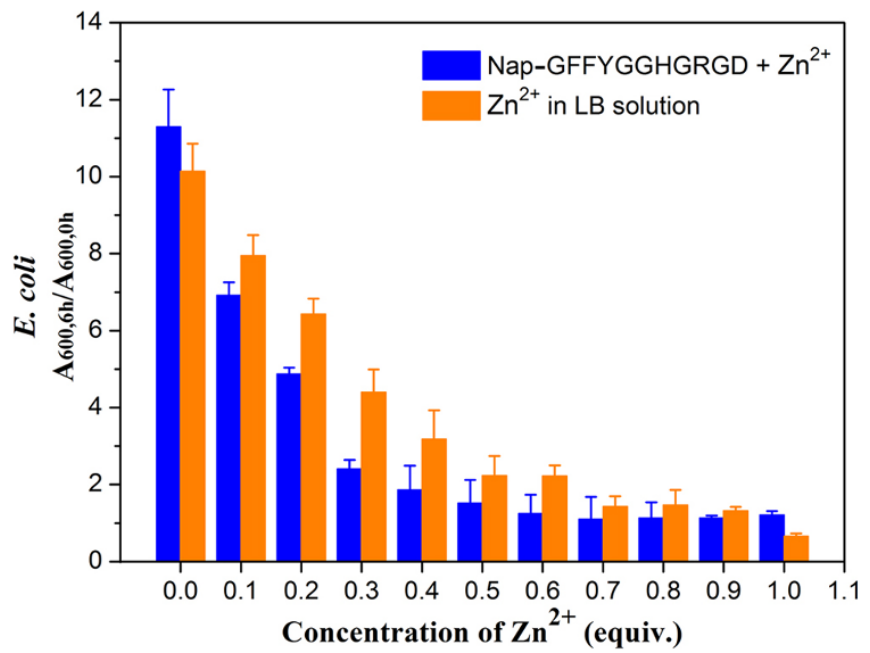

Figure 4 Growth inhibition of $E$. coli by zinc ion binding hydrogels (1.0 wt\% in LB buffer, $\mathrm{pH} 7.4$ ) and zinc ion solutions (LB buffer, $\mathrm{pH} 7.4$ ). The data is presented as the absorbance at $600 \mathrm{~nm}$ following 6 hours of growth divided by the absorbance at $600 \mathrm{~nm}$ just after the mixing of bacteria with hydrogels. Each bar represents standard error of the mean of three technical replicates.
After releasing for 6 hours, about $60 \%$ zinc ions gain freedom from the bind relationship.

Cytotoxicity study. To ensure usability of the hydrogels in biomedical applications like wound healing, the cytotoxicity of the zinc triggered hydrogel was studied on Helga cells. Results (Fig. 5A) shows that compound $\mathbf{1}$ has almost no toxicity on cells when the concentration was equal to or less than $1.0 \mathrm{wt} \%$ since more than $90 \%$ cell were alive after culturing with PBS buffer solution containing 0.1 to $1.0 \mathrm{wt} \%$ of compound 1 for 24 hours. However, when bind with zinc ions, the hydrogel become a little toxicity when concentration of zinc ion rise to higher than 0.5 equiv. and only about $70 \%$ of cells are alive (Fig. 5B).

\section{Discussion}

The fact that several divalent metal ions could not trigger hydrogelation of compound 1 along with the zeta potential results suggested that the gelation was due to the relatively specific interactions between compound 1 and metal ions. However, the charge interaction between negative peptide and positive metal ions might also play a role in hydrogelation.

According to the rheology results (Supplementary Fig. S2), though the value of $G$ ' was very low for the solution of NapGFFYGGGHGRGD, it was still larger than the G" value, which made the solution different from normal small molecular solution $\left(G^{\prime \prime} \geq\right.$ $\left.G^{\prime}\right)$. As we have discussed above, mechanical property of certain object was often affected by the structure of its components. From TEM image Fig. $3 \mathrm{~A}$ we can see that the small molecules have formed 

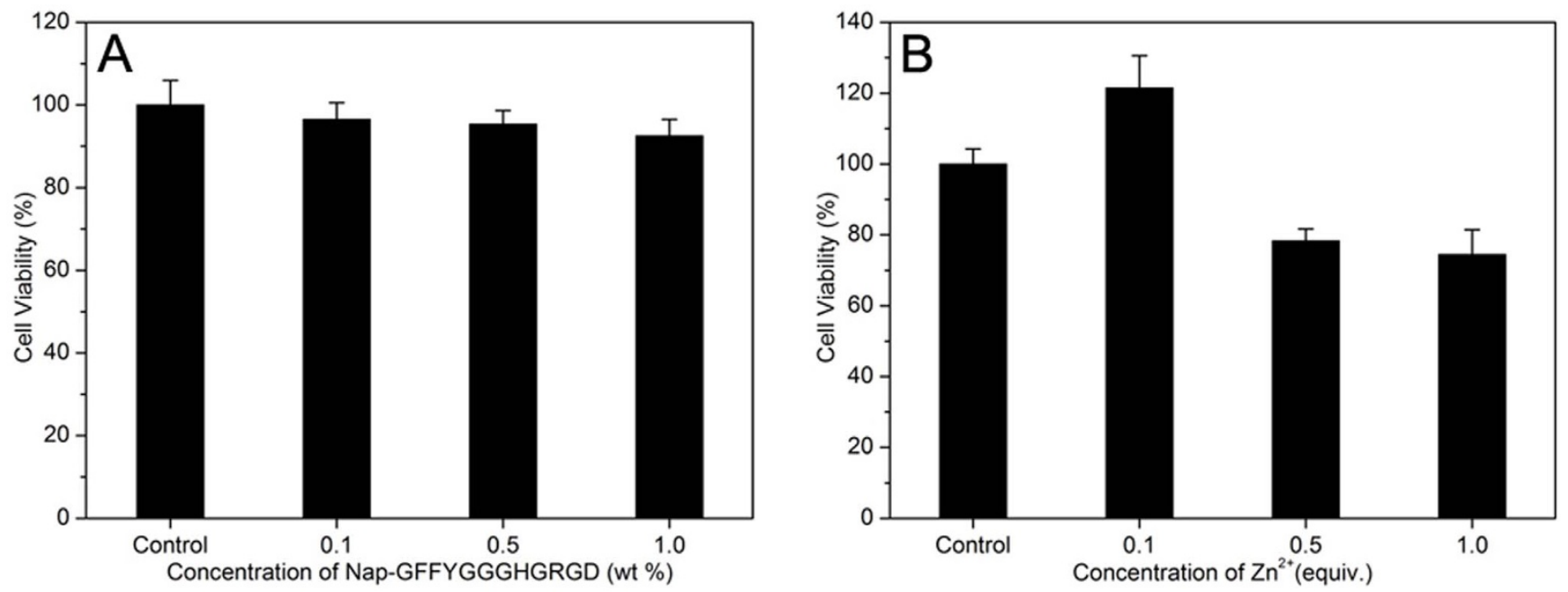

Figure 5 Cell viability assay for Hela cells. A) Relative cell viability with respect to control group (untreated cells) is plotted against increasing peptide concentrations. B) Cell viability against different amount of zinc ion in the triggered hydrogels (Concentration of peptide is $1.0 \mathrm{wt} \%$ ).

some kind of short fibers and might make the solution sticky enough to behave like some kind of weak gel in rheology. Another interesting thing is that 0.2 equiv. of $\mathrm{Zn}^{2+}$ could trigger the sol-gel transition of solutions at concentration of $1.0 \mathrm{wt} \%$, but couldn't enable the hydrogelation of solutions containing the same amount of peptide (at concentration of $0.2 \mathrm{wt} \%$ ), indicating that the already assembled short fibers in dense solutions of compound 1 might also participated into the hydrogelating process and help with the formation of hydrogels. TEM images and the rheology results have shown that more zinc ions would result in stronger fibers, thus leading to hydrogels with higher mechanical strength due to larger force bearing capacity of the fibers. Meanwhile, we noticed that the density of the fibers decreased a little while the diameter of fibers were increasing. So we came to a hypothesis that the stronger fibers were made by thinner fibers winding with each other, which could explain the reduction of fibril density while the amount of zinc binding hydrogelators was growing.

The results that zinc ion binding hydrogel have better antibacterial activity than non-encapsulated zinc ions was unexpected to us. One possible explanation of this effect is that the encapsulation of $\mathrm{Zn}^{2+}$ and subsequent release may lead to a high local concentration of $\mathrm{Zn}^{2+}$ near the bacteria. And another hypothesis is that the fibers in zinc triggered hydrogel become positive charged so that they can attract bacteria (which often bearing negative charge in LB medium) near them thus reduce the distance between bacteria and zinc ions. Even so, the reason for the enhanced antibacterial activity remains unresolved.

In hydrogels triggered by smaller amount of zinc ions (less than 0.7 equiv.), the inhibition ability against $E$. coli was clearly in concentration-dependent manner. When hydrogels containing more than 0.7 equiv. of zinc ions, the bacterial growth has been completely inhibited. During the preparation of hydrogels, we have noticed that at neutral $\mathrm{pH}$, much more zinc ions could be included in the hydrogel than in the PBS solutions. Zinc ions would precipitate in PBS solution while same amount of it could trigger the formation of hydrogels and stay homogeneously in the hydrogel. Furthermore, by increasing the concentration of compound 1 , more $\mathrm{Zn}^{2+}$ could be immobilized in the gel, hence increasing the local concentration of zinc ions around bacterial and achieve better inhibition activity. However, the increase of $\mathrm{Zn}^{2+}$ could also lead to more cytotoxicity on cells. Both aspects should be concerned when deciding the concentration of $\mathrm{Zn}^{2+}$ in the hydrogel for practical application.

In summary, our data have demonstrated that zinc ion could bind with the peptide of Nap-GFFYGGGHGRGD, and then trigger the self-assembling of this small molecule into hydrogels. The amount of $\mathrm{Zn}^{2+}$ added to the system would lead to diameter changes of nano-fibers in microscopic scale, resulting in differences on mechanical property of the hydrogel. The bacterial test have shown that the growth of $E$. coli cultured within our hydrogel is inhibited in 6 hours, confirming the antibacterial activity of our zinc ion triggered small molecular hydrogel. It is a very versatile system because i) other bioactive peptide (e.g. antibacterial peptides) can be coded into the peptide sequence; ii) other antibacterial agents can be loaded in the hydrogel together with the zinc ions, including many bioactive molecules for the good biocompatibility of peptide-based hydrogels; iii) both PBS, LB and DMEM medium (Supplementary Fig. S6) could be gelated. Further in vivo experiments about this hydrogel is on going for the final purpose of successfully clinical application.

\section{Methods}

Synthesis and Purification of Nap-GFFYGGGHGRGD (1). Nap-

GFFYGGGHGRGD was synthesized according to solid-phase peptide synthesis (SPPS) methods as described in the supplementary method. The crude product was directly separated by HPLC with MeOH-water as the eluents and the target product was collected. As shown in Supplementary Fig. S2A, there is only one narrow single peak on the LC-MS spectrum indicating the target product was very pure. Characterization of the peptide including ${ }^{1} \mathrm{H}$ NMR spectrum (Supplementary Fig. S4), ESI-MS calculated m/z: 1393.58 [(M + H $)^{2+}$, expected: 1395.85] (Supplementary Fig. S2B) and HR-MS calculated m/z: $1393.5840\left[(\mathrm{M}+\mathrm{H})^{+}\right.$, expected: 1394.5875] (Supplementary Fig. S3) have proved that pure Nap-GFFYGGGHGRGD was obtained.

Preparation of Hydrogels. In order to test the ability of hydrogelation of NapGFFYGGGHGRGD, the peptide was first dissolved in a small amount of solvents (PBS, LB or DMEM Medium), then sodium carbonate solution were added to adjust the $\mathrm{pH}$ to about 7.4 to form a stock solution. Afterward, the stock solution was diluted into desire concentration in a small tube, with addition of acidic solution containing different equiv. of $\mathrm{ZnCl}_{2}$. The final solution were adjusted to neutral $\mathrm{pH}$ with sodium carbonate and the small tube was then placed up-side-down to test whether the hydrogel was formed. Optical images of hydrogels was taken by digital camera (Supplementary Fig. S6) and minimum hydrogelation concentration of the peptide and zinc ions data was in Table S1.

Rheology Test. Dynamic frequency and strain sweep rheology experiments were performed on a TA instruments rheometer (AR 2000ex) system using a $40 \mathrm{~mm}$ stainless steel parallel-plate at the gap of $500 \mu \mathrm{m}$. Hydrogels were firstly prepared according to above mentioned procedure. The hydrogel sample was added to the rheometer pre-equilibrated at $20^{\circ} \mathrm{C}$ for about 1 hour. Dynamic frequency sweep experiments $\left(0.1\right.$ to $\left.100 \mathrm{rad} \mathrm{s}^{-1}\right)$ were run at the same temperature and strain were maintained at $0.1 \%$. A dynamic strain sweep (0.1 to $10 \%$ strain) was performed afterward in which the temperature and frequency were maintained at $20^{\circ} \mathrm{C}$ and $2 \mathrm{rad}$ $\mathrm{s}^{-1}$, respectively. Measurements were performed in triplicate. Rheology results of each sample were shown in Supplementary Fig. S8.

Transmission Electron Microscopy. A small drop of diluted gels was placed on a copper TEM grid (20-30 nm film deposit of pure carbon). 30 seconds later, the water 
on the edge of the grid was dried by a filter paper. Then a drop of staining solution (1.0 $\mathrm{wt} \%$ aqueous uranyl acetate) was immediately added to the grid. After setting for 10 seconds, the stain was blotted off carefully with filter paper. Images were taken in bright-field mode with a Tecnai G2 F20 system, operating at $200 \mathrm{kV}$ accelerating voltage.

Bacterial Inhibition Test. Nap-GFFYGGGHGRGD was dissolved in LB medium and the $\mathrm{pH}$ was adjusted to 7.4 to obtain a stock solution in the concentration of 2.0 wt\%. $50 \mu \mathrm{l}$ of stock solution was added to different on a sterile 96 well plates, then $45 \mu \mathrm{l}$ of solution containing different amount of $\mathrm{ZnCl}_{2}$ were added to the holes to form a series of hydrogels. $5 \mu \mathrm{L}$ bacterial solution with $\mathrm{OD}_{600}$ value of 0.6 was added immediately to each hole and mixed with the hydrogel gently, achieved $100 \mu \mathrm{l}$ mixture of bacteria and hydrogel at final concentration of $1.0 \mathrm{wt} \% .5 \mu \mathrm{L}$ bacterial solution with $\mathrm{OD}_{600}$ value of 0.6 was added to holes containing $\mathrm{LB}$ medium only were set to be control group. The plates were incubated at $37^{\circ} \mathrm{C}$ for 6 hours, and the absorbance at wavelength of $600 \mathrm{~nm}$ was measured. Each measurement was performed in triplicate. Similar procedure was used to evaluate the antibacterial ability of LB medium and the hydrogel of Nap-FFGRGD. For the antibacterial test on surface of hydrogel, $800 \mu \mathrm{L}$ of hydrogels was formed in the hole of 24 well plates. In order to gain steady hydrogels, we keep the plates standing at room for 24 hour, then $1 \mathrm{~mL}$ of LB medium were added to each holes and removed after 5 mins. Bacterial solution with $\mathrm{OD}_{600}$ value of 0.6 were diluted by ten thousand times and smeared onto the surface of each gel evenly, then the 24 plates was placed upside down in the $37^{\circ} \mathrm{C}$ incubator for 24 hours to see if the bacteria grew into colonies.

Cytotoxicity Study. The cytotoxicity of the compounds were evaluated by Cell Counting Kit (CCK-8) assay. Hela cells were seeded into 96 -well plates at a density of 10,000 cells per-well and incubated for $24 \mathrm{~h}$. The powder resulting from freeze-dried hydrogels or peptides were dissolved in DMEM (Dulbecco's Modified Eagle Medium) solutions, and $200 \mu \mathrm{L}$ above solutions were added into the cells. After incubation for another 24 hour, $10 \mu \mathrm{L}$ of CCK-8 solution was added to each well. Four hours later, the plates were directly taken to measure the optical density of solutions at $450 \mathrm{~nm}$ using a microplate reader (Labsystem, Multiskan, Ascent, Finland). The Hela cell without any treatments were used as the control and its cell viability were set to $100 \%$. Each experiment was repeated 3 times.

1. Haley, R. W. et al. Nosocomial infections in U.S. hospitals, 1975-1976: Estimated frequency by selected characteristics of patients. Am. J. Med. 70, 947-959 (1981).

2. Klevens, R. M. et al. Estimating health care-associated infections and deaths in U.S. Hospitals, 2002. Public Health Rep. 122, 160-166 (2007).

3. Lipsky, B. A., Itani, K., Norden, C. \& Linezolid Diabet Foot Infect, S. Treating foot infections in diabetic patients: A randomized, multicenter, open-label trial of linezolid versus ampicillin-sulbactam/amoxicillin-clavulanate. Clin. Infect. Dis. 38, 17-24 (2004).

4. Hajipour, M. J. et al. Antibacterial properties of nanoparticles. Trends Biotechnol. 30, 499-511 (2012)

5. Jayaraman, R. Antibiotic resistance: An overview of mechanisms and a paradigm shift. Curr. Sci. 96, 1475-1484 (2009).

6. Deurenberg, R. H. \& Stobberingh, E. E. The Molecular Evolution of Hospital- and Community-Associated Methicillin-Resistant Staphylococcus aureus. Curr. Mol. Med. 9, 100-115 (2009).

7. Mah, T. F. C. \& O’Toole, G. A. Mechanisms of biofilm resistance to antimicrobial agents. Trends Microbiol. 9, 34-39 (2001).

8. Davies, D. Understanding biofilm resistance to antibacterial agents. Nat. Rev. Drug Discovery 2, 114-122 (2003).

9. Xu, K. D., McFeters, G. A. \& Stewart, P. S. Biofilm resistance to antimicrobial agents. Microbiology-Uk 146, 547-549 (2000)

10. Pfaller, M. A. \& Diekema, D. J. Epidemiology of invasive candidiasis: a persistent public health problem. Clin. Microbiol. Rev. 20, 133-+ (2007)

11. Bendouah, Z., Barbeau, J., Abou Hamad, W. \& Desrosiers, M. Biofilm formation by Staphylococcus aureus and Pseudomonas aeruginosa is associated with an unfavorable evolution after surgery for chronic sinusitis and nasal polyposis. Otolaryngol. Head Neck Surg. 134, 991-996 (2006).

12. Fihn, S. D., Johnson, C. \& Stamm, W. E. Escherichia coli urethritis in women with symptoms of acute urinary tract infection. J. Infect. Dis. 157, 196-199 (1988).

13. Idress, M., Mussarat, U., Badshah, Y., Qamar, R. \& Bokhari, H. Virulence factors profile of drug-resistant Escherichia coli isolates from urinary tract infections in Punjab, Pakistan. Eur. J. Clin. Microbiol. Infect. Dis. 29, 1533-1537 (2010).

14. Borrero, N. V. et al. Phenazine antibiotic inspired discovery of potent bromophenazine antibacterial agents against Staphylococcus aureus and Staphylococcus epidermidis. Org. Biomol. Chem. 12, 881-886 (2014).

15. Dubin, A. et al. New generation of peptide antibiotics. Acta Biochim. Pol. $\mathbf{5 2}$ 633-638 (2005).

16. Yang, Z., Liang, G., Guo, Z., Guo, Z. \& Xu, B. Intracellular hydrogelation of small molecules inhibits bacterial growth. Angew. Chem. Int. Ed. Engl. 46, 8216-8219 (2007).

17. Huh, A. J. \& Kwon, Y. J. "Nanoantibiotics": A new paradigm for treating infectious diseases using nanomaterials in the antibiotics resistant era. J. Control. Release 156, 128-145 (2011)
18. James, C., Johnson, A. L. \& Jenkins, A. T. A. Antimicrobial surface grafted thermally responsive PNIPAM-co-ALA nano-gels. Chem. Commun. 47, 12777-12779 (2011)

19. Fernández, G. A., Vela Gurovic, M. S., Olivera, N. L., Chopa, A. B. \& Silbestri, G. F. Antibacterial properties of water-soluble gold(I) N-heterocyclic carbene complexes. J. Inorg. Biochem. 135, 54-57 (2014).

20. Kaariainen, M. L. et al. Zinc release from atomic layer deposited zinc oxide thin films and its antibacterial effect on Escherichia coil. Appl. Surf. Sci. 287, 375-380 (2013).

21. Park, J-S. et al. Synthesis and characterization of zinc chloride containing poly(acrylic acid) hydrogel by gamma irradiation. Radiat. Phys. Chem. 88, 60-64 (2013).

22. Ren, G. et al. Characterisation of copper oxide nanoparticles for antimicrobial applications. Int. J. Antimicrob. Agents 33, 587-590 (2009).

23. Maness, P. C. et al. Bactericidal activity of photocatalytic $\mathrm{TiO}(2)$ reaction: toward an understanding of its killing mechanism. Appl. Environ. Microbiol. 65, 4094-4098 (1999).

24. Shunmugaperumal, T. \& Ramamurthy, S. Antibiofilm activity of nanosized magnesium fluoride (vol 30, pg 5969, 2009). Drug Dev. Ind. Pharm. 38, 899-899 (2012).

25. Taheri, S. et al. Synthesis and antibacterial properties of a hybrid of silver-potato starch nanocapsules by miniemulsion/polyaddition polymerization. J. Mater. Chem. B Mater. Biol. Med. 2, 1838-1845 (2014).

26. Dai, Y. et al. Up-Conversion Cell Imaging and pH-Induced Thermally Controlled Drug Release from NaYF4:Yb3+/Er3+@Hydrogel Core-Shell Hybrid Microspheres. ACS Nano 6, 3327-3338 (2012).

27. Zhu, Z. et al. An Aptamer Cross-Linked Hydrogel as a Colorimetric Platform for Visual Detection. Angew. Chem. Int. Ed. Engl. 49, 1052-1056 (2010).

28. Zhao, L., Weir, M. D. \& Xu, H. H. K. An injectable calcium phosphate-alginate hydrogel-umbilical cord mesenchymal stem cell paste for bone tissue engineering. Biomaterials 31, 6502-6510 (2010).

29. Murakami, K. et al. Hydrogel blends of chitin/chitosan, fucoidan and alginate as healing-impaired wound dressings. Biomaterials 31, 83-90 (2010).

30. Gao, J. et al. Enzyme-controllable delivery of nitric oxide from a molecular hydrogel. Chem. Commun. 49, 9173-9175 (2013).

31. Zhao, Q. et al. Polysaccharide-based biomaterials with on-demand nitric oxide releasing property regulated by enzyme catalysis. Biomaterials 34, 8450-8458 (2013).

32. Malzahn, K. et al. Advanced dextran based nanogels for fighting Staphylococcus aureus infections by sustained zinc release. J. Mater. Chem. B Mater. Biol. Med. 2, 2175-2183 (2014).

33. Pelgrift, R. Y. \& Friedman, A. J. Nanotechnology as a therapeutic tool to combat microbial resistance. Adv. Drug Delivery Rev. 65, 1803-1815 (2013).

34. Buhrman, J. S., Cook, L. C., Rayahin, J. E., Federle, M. J. \& Gemeinhart, R. A. Proteolytically activated anti-bacterial hydrogel microspheres. J. Control. Release 171, 288-295 (2013).

35. Rasale, D. B., Maity, I., Konda, M. \& Das, A. K. Peptide self-assembly driven by oxo-ester mediated native chemical ligation. Chem. Commun. 49, 4815-4817 (2013).

36. Liu, G. F., Zhang, D. \& Feng, C. L. Control of three-dimensional cell adhesion by the chirality of nanofibers in hydrogels. Angew. Chem. Int. Ed. Engl. 53, 7789-7793 (2014)

37. Li, P., Yin, Z., Dou, X. Q., Zhou, G. \& Feng, C. L. Convenient three-dimensional cell culture in supermolecular hydrogels. ACS Appl. Mater. Interfaces 6, 7948-7952 (2014).

38. Ray, S., Das, A. K. \& Banerjee, A. pH-Responsive, Bolaamphiphile-Based Smart Metallo-Hydrogels as Potential Dye-Adsorbing Agents, Water Purifier, and Vitamin B12 Carrier. Chem. Mater. 19, 1633-1639 (2007).

39. Basak, S., Nanda, J. \& Banerjee, A. Multi-stimuli responsive self-healing metallohydrogels: tuning of the gel recovery property. Chem. Commun. 50, 2356-2359 (2014).

40. Roy, S., Baral, A. \& Banerjee, A. An amino-acid-based self-healing hydrogel: modulation of the self-healing properties by incorporating carbon-based nanomaterials. Chemistry 19, 14950-14957 (2013).

41. Chen, L. et al. Salt-induced hydrogelation of functionalised-dipeptides at high $\mathrm{pH}$ Chem. Commun. 47, 12071-12073 (2011)

42. Gao, J. et al. Enzyme promotes the hydrogelation from a hydrophobic small molecule. J. Am. Chem. Soc. 131, 11286-11287 (2009).

43. Yang, Z., Liang, G. \& Xu, B. Enzymatic hydrogelation of small molecules. Acc. Chem. Res. 41, 315-326 (2008)

44. Debnath, S., Roy, S. \& Ulijn, R. V. Peptide Nanofibers with Dynamic Instability through Nonequilibrium Biocatalytic Assembly. J. Am. Chem. Soc. 135 16789-16792 (2013).

45. Nalluri, S. K. M., Berdugo, C., Javid, N., Frederix, P. W. J. M. \& Ulijn, R. V. Biocatalytic Self-Assembly of Supramolecular Charge-Transfer Nanostructures Based on n-Type Semiconductor-Appended Peptides. Angew. Chem. Int. Ed. Engl. 53, 5882-5887 (2014).

46. Yu, H. et al. Photoinduced Tandem Reactions of Isoquinoline-1,3,4-trione with Alkynes To Build Aza-polycycles. J. Org. Chem. 75, 2989-3001 (2010).

47. Cao, W., Zhang, X., Miao, X., Yang, Z. \& Xu, H. $\gamma$-Ray-Responsive Supramolecular Hydrogel Based on a Diselenide-Containing Polymer and a Peptide. Angew. Chem. Int. Ed. Engl. 52, 6233-6237 (2013). 
48. Yoshii, T., Ikeda, M. \& Hamachi, I. Two-photon-responsive supramolecular hydrogel for controlling materials motion in micrometer space. Angew. Chem. Int. Ed. Engl. 53, 7264-7267 (2014).

49. Raeburn, J., McDonald, T. O. \& Adams, D. J. Dipeptide hydrogelation triggered via ultraviolet light. Chem. Commun. 48, 9355-9357 (2012).

50. Ikeda, M. et al. Installing logic-gate responses to a variety of biological substances in supramolecular hydrogel-enzyme hybrids. Nat. Chem. 6, 511-518 (2014).

51. Rasale, D. B., Maity, I. \& Das, A. K. In situ generation of redox active peptides driven by selenoester mediated native chemical ligation. Chem. Commun. 50, 11397-11400 (2014)

52. Miao, X. et al. Switchable Catalytic Activity: Selenium-Containing Peptides with Redox-Controllable Self-Assembly Properties. Angew. Chem. Int. Ed. Engl. 52, 7781-7785 (2013).

53. Spoerke, E. D., Anthony, S. G. \& Stupp, S. I. Enzyme Directed Templating of Artificial Bone Mineral. Adv. Mater. 21, 425-430 (2009).

54. Knerr, P. J., Branco, M. C., Nagarkar, R., Pochan, D. J. \& Schneider, J. P. Heavy metal ion hydrogelation of a self-assembling peptide via cysteinyl chelation. J. Mater. Chem. 22, 1352-1357 (2012).

55. Micklitsch, C. M. et al. Zinc-Triggered Hydrogelation of a Self-Assembling betaHairpin Peptide. Angew. Chem. Int. Ed. Engl. 50, 1577-1579 (2011).

56. Shi, J., Gao, Y., Zhang, Y., Pan, Y. \& Xu, B. Calcium Ions to Cross-Link Supramolecular Nanofibers to Tune the Elasticity of Hydrogels over Orders of Magnitude. Langmuir 27, 14425-14431 (2011).

57. Pan, Y., Gao, Y., Shi, J., Wang, L. \& Xu, B. A versatile supramolecular hydrogel of nitrilotriacetic acid (NTA) for binding metal ions and magnetorheological response. J. Mater. Chem. 21, 6804-6806 (2011).

58. Kuang, Y., Gao, Y., Shi, J., Lin, H.-C. \& Xu, B. Supramolecular hydrogels based on the epitope of potassium ion channels. Chem. Commun. 47, 8772-8774 (2011).

59. Mei, J. et al. Barium-triggered beta-sheet formation and hydrogelation of a short peptide derivative. Rsc Advances 4, 1193-1196 (2014).
60. Gokhale, N. H., Bradford, S. \& Cowan, J. A. Catalytic inactivation of human carbonic anhydrase I by a metallopeptide-sulfonamide conjugate is mediated by oxidation of active site residues. J. Am. Chem. Soc. 130, 2388-2389 (2008).

\section{Acknowledgments}

This work was supported by the Fundamental Research Funds for the Central Universities (Grant No. 65141007), National Natural Science Foundation of China (Grant No. $81302082,81272685,31301151$ and 81172355), Key Program of Natural Science Foundation of Tianjin (Grant No. 11JCZDJC18400 and 13YCYBYC37400) and Major Anticancer Technologies R \& D Program of Tianjin (Grant No. 12ZCDZSY16700).

\section{Author contributions}

J.G. and J.H. designed the project and wrote the manuscript. C.X., Y.C. and C.R. did the syntheses, characterizations and bacterial experiments. All authors helped with manuscript preparation and revision.

\section{Additional information}

Supplementary information accompanies this paper at http://www.nature.com/ scientificreports

Competing financial interests: The authors declare no competing financial interests.

How to cite this article: Xu, C., Cai, Y., Ren, C., Gao, J. \& Hao, J. Zinc-Triggered Hydrogelation of Self-assembled Small Molecules to Inhibit Bacterial Growth. Sci. Rep. 5, 7753; DOI:10.1038/srep07753 (2015)

This work is licensed under a Creative Commons Attribution-NonCommercialNoDerivs 4.0 International License. The images or other third party material in this article are included in the article's Creative Commons license, unless indicated otherwise in the credit line; if the material is not included under the Creative Commons license, users will need to obtain permission from the license holder in order to reproduce the material. To view a copy of this license, visit http:// creativecommons.org/licenses/by-nc-nd/4.0/ 